\title{
Examination of the bus lane enforcement system in Thessaloniki
}

\author{
S. Basbas \\ Department of Transportation and Hydraulic Engineering, \\ Faculty of Rural and Surveying Engineering, School of Technology, \\ Aristotle University of Thessaloniki, Greece
}

\begin{abstract}
One way to deal with the traffic and associated environmental problems in urban areas is the promotion of Public Transport. This can be achieved, among other things, through the implementation of bus priority measures. Bus lanes comprise the most widespread measure worldwide and their success mainly depends on the efficiency of the enforcement. The international experience shows that enforcement is mainly based nowadays on stationary and bus mounted cameras. Within the framework of this paper the existing situation, as far as the bus lanes violators in the city of Thessaloniki are concerned, is presented. Results are based on a survey that was carried out in December 2005 and these results were compared to the ones obtained from previous surveys in the same area. The vast majority of violators concern the drivers of two-wheel cycles followed by taxi drivers. In some cases drivers of the two-wheel cycles can legally enter the bus lanes (something which is foreseen in the Greek Highway Code under certain circumstances), then taxi drivers consist of more than half of the violators of the bus lane network.
\end{abstract}

Keywords: bus lane, enforcement, bus priority, stationary cameras.

\section{Introduction}

Bus priority measures play a very important role in the efforts of local authorities all over the world in order to promote the use of Public Transport and at the same time to reduce traffic congestion as well as the associated environmental impacts. The reliability of the Public Transport system is improved as a result of the bus priority measures and this fact encourage people to use buses instead of 
passenger cars, something which leads to an overall improvement of the environmental conditions and the quality of life. Bus priority measures include [1] with-flow and contra-flow bus lanes, bus gates and rising bollards, guided busways, pre-signals and bus advanced areas, Selective Vehicle Detection (SVD), MOVA, busSCOOT, Automatic Vehicle Location (AVL), mixed priority streets and bus friendly traffic calming (green), High Occupancy Vehicle (HOV) lanes and no-car lanes, Bus Park and Ride and complementary measures. Bus lanes are one of the most widespread measures and enforcement is of great importance for their success. Bus lanes enforcement systems include bus mounted cameras, CCTV cameras and fixed cameras. As an example of the first case it is mentioned that the MVA Consultancy design and implement a trial system for the city of Birmingham [2]. The bus lane enforcement system consisted of video cameras and vision processing equipment. The system was capable of taking digital photographs of vehicles $20 \mathrm{~m}$ ahead of the bus and to transmit the registration number of the vehicles to a VMS near the end of the bus lane. Another bus mounted cameras system is the JAI Bus Lane Enforcement Cameras consisted of 2 CCTV cameras [3]. The JAI cameras are built into the London buses and record twin images on to a video. Many municipalities in various countries around the world have undertaken bus lane camera enforcement based on specific regulations. For example regulations made under section 144 of the Transport Act 2000 which came into force on 1 November 2005, allow approved County Councils, Metropolitan District Councils and Unitary Authorities in England to enforce their bus lanes [4]. In 1997, London launched a bus lane camera enforcement using bus mounted cameras and stationary cameras. In the year 2004 a number of 900 cameras on buses and 500 roadside cameras for 700 bus lanes and as a consequence, bus use was up 7\% and reliability $12.5 \%$ [5]. Manchester City Council enforce bus lanes from 22 September 2006 and as a consequence if a vehicle is captured by CCTV using a bus lane during restricted hours a Penalty Charge Notice (PCN) may be issued [6]. Brighton \& Hove City Council also launched bus lane camera enforcement from 15 March 2007 [7]. The PCN for motorist who ignore the bus lanes signs is $£ 60$, reduced to $£ 30$ if paid within 14 days. It is also important to notice that “in January 2004 Scotland's first Bus Lane Enforcement Camera system became operational in Aberdeen" [8]. A number of 8 cameras were installed at problematic locations and the system is operated by Grampian Police in partnership with the City Council. As far as the efficiency of the system is concerned, recent figures would suggest that as few as $10 \%$ of offenders are being issued with fixed penalty notice. Bus lane cameras are also into operation in various locations in NSW, Australia (i.e., Darlinghurst, Paddington, Sydney, Ultimo, Glebe, Smithfield, Prairewood, Bonnyrigg, Hinchnbrook, Liverpool) [9]. The Chicago Transit Authority began the bus lane enforcement camera pilot in June 2004 and then began issuing citations in August 2005 [10]. In San Francisco, the cost for bus lane violators caught on cameras installed on Muni buses is proposed to be $\$ 100$ [11]. Within the framework of this paper the examination of the bus lane enforcement system in the city of Thessaloniki, Greece, is presented. The analysis of the bus lane violators as far as certain 
criteria (i.e., vehicle type, length, duration) are concerned is also presented. Finally the analysis results are compared to the results of previous studies in the same area.

\section{Presentation of the bus lane system in the city of Thessaloniki}

Thessaloniki, the second biggest city in Greece, is situated in the north part of the country and has a population of around one million inhabitants. Nowadays the public transport system in the city consists only of buses. The Thessaloniki Bus Operator (O.A.S.Th.) has a total number of 583 buses (of which 279 are articulated) [12]. There are 72 bus lines carrying around 150 million passengers per year (that means around 12.500 .000 passengers/month or 411.000 passengers/day). There are four with-flow bus lanes in the city, three of which are found in the central area. The first with-flow bus lane was implemented in the Mitropoleos Rd. (one-way street in the city centre) in the early 90s and has a length of $980 \mathrm{~m}$. The second with-flow bus lane was implemented in Vas.Olgas Rd. (one way street) in 1992. Initially it had a total length of $3.200 \mathrm{~m}$. and recently (2nd half of 2006) it has been extended towards its eastern part. In 1996 a third with-flow bus lane was implemented in the central area, and more especially in the Tsimiski Rd. (one way street). The length of the third bus lane is $1.300 \mathrm{~m}$. The last with-flow bus lane was implemented in Egnatia RdMonastiriou Rd axis (city centre) and has a length of $1750 \mathrm{~m}$. (This axis is a twoway street). Although the benefits in term of bus speed were very significant immediately after the implementation of the bus lanes, these benefits tend to be decreased nowadays due to the bus lane violations. Originally the enforcement was made with police cars or motorcycles. This presupposes a substantial number of police staff to be assigned to enforcement duties, something which is not easy on a daily basis due to the extensive length of the bus lane network. This is the reason why it was decided to implement a bus lane camera enforcement system using stationary cameras. The existing system consists of 5 ordinary cameras (with film) and 5 digital cameras (inductive loops are installed under the pavement where cameras are placed). The results obtained from the system are presented in Table 1.

Table 1: Violators according to the bus lane camera enforcement system.

\begin{tabular}{|l|c|c|c|}
\hline \multicolumn{1}{|c|}{ Time period } & Taxi & Other vehicles & Total \\
\hline $18-6-2004$ to $31-12-2004$ & 717 & 1417 & 2134 \\
\hline $1-1-2005$ to $31-12-2005$ & 553 & 1163 & 1716 \\
\hline $1-1-2006$ to $6-2-2006$ & 38 & 43 & 81 \\
\hline
\end{tabular}

Source: [13].

It is important to mention at this point that according to the Highway Code (which will come into force on the $3^{\text {rd }}$ of June, 2007) five points in the point system are foreseen for the violation of bus lanes (a total number of 25 points lead to loss of the driving license). A penalty charge of 200 euros is also foreseen 
for the bus lane violators. Although public transport buses are only allowed to use the bus lanes, two wheel cycles can also use them if a traffic study suggests it. The authorities have recently decided (Spring 2007) to allow taxi drivers to use the bus lanes due to the fact that a metro system is under construction in the central area of the city. As a consequence, the number of lanes on main roads dedicated to serve passengers cars, taxi etc. is reduced and thus the authorities would like to facilitate the taxis. Photographs of the existing bus lane network in the city of Thessaloniki are presented in Figure 1.

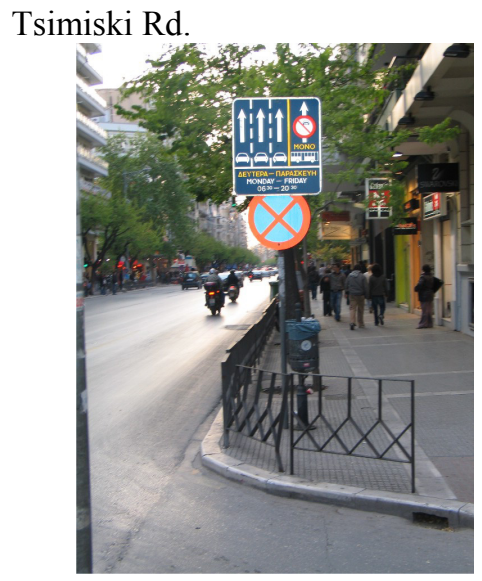

Mitropoleos Rd.

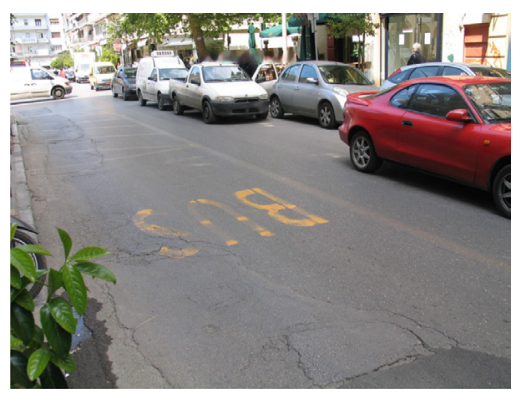

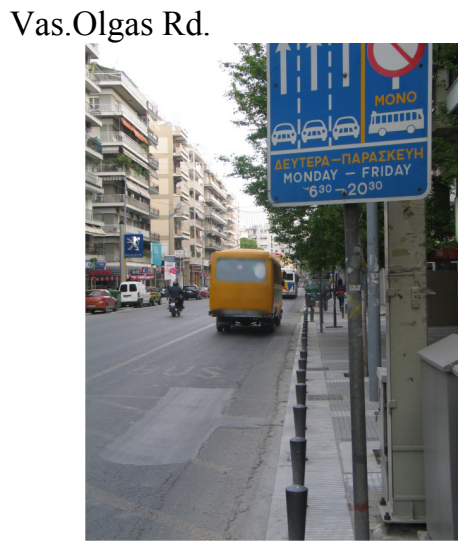

Egnatia Rd.

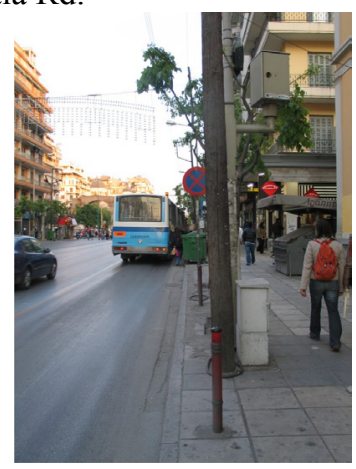

Figure 1: $\quad$ Bus lanes in the Thessaloniki city network.

\section{Examination of the bus lane violations}

The survey concerning the examination of bus lanes violations in the city of Thessaloniki was carried out in the framework of the research activities of the Department of Transportation \& Hydraulic Engineering, Faculty of Rural \& Surveying Engineering, Aristotle University of Thessaloniki (AUTh) in December 2005 [14]. Observations were made on the $9^{\text {th }}$ of December and cover the following periods: 
- Egnatia Rd. (East-West direction) 08:00-09:30 \& 17:00-18:30

- $\quad$ Egnatia Rd. (West-East direction) 14:00-15:00 \& 18:30-20:00

- Tsimiski Rd. 08:00-09:30 \& 17:00-18:30

- Mitropoleos Rd. 14:00-15:30 \& 18:30-20:00

Five vehicle types were considered for the purposes of the specific survey, namely two wheel cycles, passenger cars, taxi, Heavy Goods Vehicles (HGV), buses (other than Public Transport buses) and state cars (cars belonging to various Public Services). The results obtained per vehicle type are presented in Table 2.

Table 2: $\quad$ Number of bus lane violations by vehicle type.

\begin{tabular}{|l|l|l|l|l|l|l|l|l|}
\hline Bus lane & $\begin{array}{l}\text { Peak } \\
\text { period }\end{array}$ & $\begin{array}{l}\text { Two } \\
\text { wheel } \\
\text { cycles }\end{array}$ & Cars & Taxi & HGV & Buses & $\begin{array}{l}\text { State } \\
\text { cars }\end{array}$ & Total \\
\hline \multirow{2}{*}{$\begin{array}{l}\text { Egnatia } \\
(\text { E-W) }\end{array}$} & Morning & 253 & 27 & 43 & 0 & 5 & 9 & 337 \\
\cline { 2 - 10 } & Afternoon & 177 & 65 & 67 & 4 & 2 & 3 & 316 \\
\cline { 2 - 10 } & Average & 215 & 46 & 55 & 2 & 4 & 6 & 327 \\
\hline \multirow{2}{*}{$\begin{array}{l}\text { Egnatia } \\
(\text { W-E) }\end{array}$} & Noon & 148 & 29 & 69 & 1 & 6 & 0 & 253 \\
\cline { 2 - 10 } & Night & 95 & 70 & 126 & 2 & 2 & 14 & 296 \\
\cline { 2 - 9 } & Average & 122 & 50 & 98 & 2 & 4 & 7 & 275 \\
\hline \multirow{5}{*}{ Tsimiski } & Morning & 702 & 36 & 73 & 1 & 10 & 18 & 840 \\
\cline { 2 - 9 } & Afternoon & 359 & 60 & 60 & 0 & 0 & 13 & 492 \\
\cline { 2 - 9 } & Average & 531 & 48 & 67 & 1 & 5 & 16 & 666 \\
\hline \multirow{5}{*}{ Mitropoleos } & Noon & 55 & 94 & 145 & 8 & 6 & 3 & 311 \\
\cline { 2 - 9 } & Night & 46 & 82 & 252 & 2 & 0 & 1 & 383 \\
\cline { 2 - 9 } & Average & 51 & 88 & 199 & 5 & 3 & 2 & 347 \\
\cline { 2 - 9 } & Morning & 955 & 63 & 116 & 1 & 15 & 27 & 1177 \\
\cline { 2 - 9 } & Noon & 203 & 123 & 214 & 9 & 12 & 3 & 564 \\
\cline { 2 - 9 } & Afternoon & 536 & 125 & 127 & 4 & 2 & 16 & 808 \\
\cline { 2 - 9 } & Night & 141 & 152 & 378 & 4 & 2 & 15 & 679 \\
\cline { 2 - 8 } & Average & 459 & 116 & 209 & 5 & 8 & 15 & 807 \\
\hline
\end{tabular}

The violations made by all types of vehicles, expressed as a percentage, are presented in Figure 2. It must be mentioned at this point that the first graph in Figure 2 corresponds to the situation where drivers of all types of vehicles are considered as possible bus lane violators. The second graph corresponds to the situation where drivers of two-wheel cycles are excluded, since it was assumed that they are entitled to legally enter the bus lanes. In the first case two-wheel cyclists consist more than half of the total violators followed by drivers of taxi and passenger cars. In the second case taxi drivers consist the vast majority of violators.

The violations made by vehicle type in each observation period, expressed as percentages, are presented in Figure 3. The period of the day is strongly associated with the type of the vehicle. Two-wheel cycles mostly appear during the morning peak. HGVs mostly appear during noon peak while (other than PT) buses appear during morning peak (i.e. school buses etc.). Passenger cars are more or less equally distributed during noon, afternoon and night peak. 


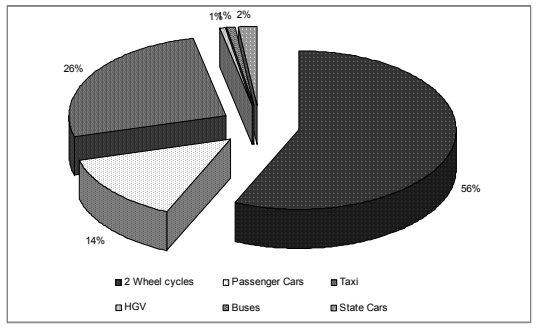

Violations - all types of vehicles

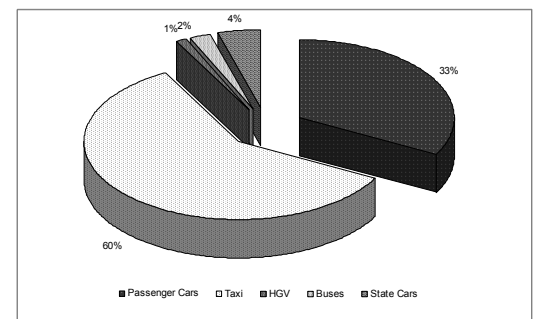

Violations- 2-wheel cycles excluded

Figure 2: $\quad$ Bus lane violations made by vehicle type.

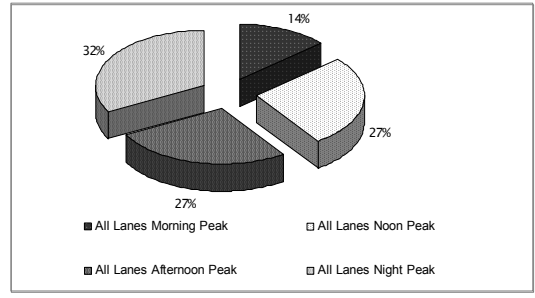

Violations made by Passenger Cars

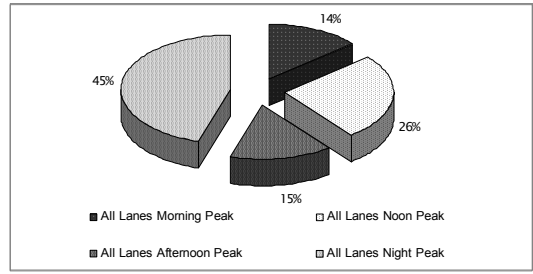

Violations made by Taxi

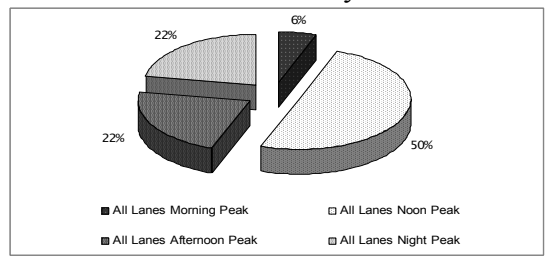

Violations made by HGV

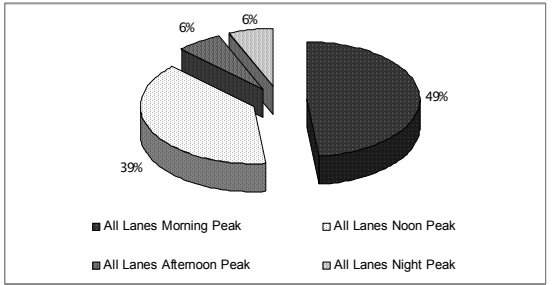

Violations made by Buses

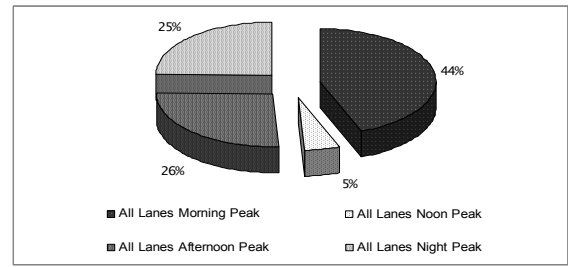

Violations made by State Cars

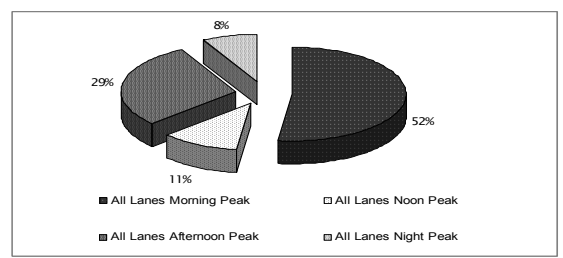

Violations made by 2-wheel cycles

Figure 3: Bus lane violations made by vehicle type in each observation period.

Bus lane violations are also analysed as far as the duration of violation for each vehicle is concerned. Six time zones were considered for the purposes of the survey (0-15 sec, 16-30 sec, 31-45 sec, 45-60 sec, 61-120 sec and 121-130 $\mathrm{sec})$. The results obtained are presented in Figure 4. As it was highly expected, the vast majority of violations last for less than $15 \mathrm{sec}$. This can be possible explained by the fact that drivers violate the bus lanes at road sections which are between the locations of the cameras and outside their range. Bus lane violations 
are also analysed as far as the length of the violation for each vehicle is concerned. Six intervals were considered for the purposes of the survey $(0-20 \mathrm{~m}$, 21-40 m, 41-60 m, 61-80 m, 81-100 m, >100 m). The results obtained are presented in Figure 5. It should be noticed that drivers usually drive at a high speed when they violate the bus lane in order to minimize the time for which they are exposed to the enforcement system.

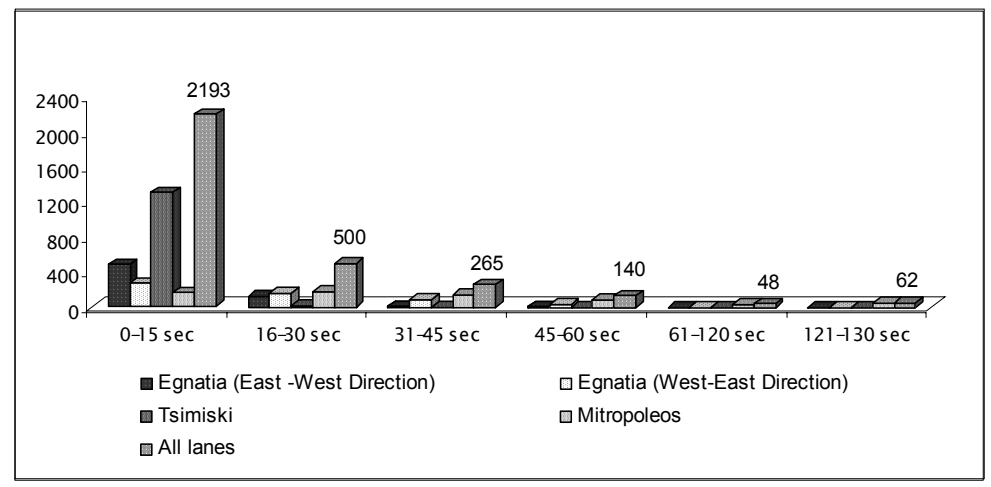

Figure 4: Bus lane violations per duration of violation.

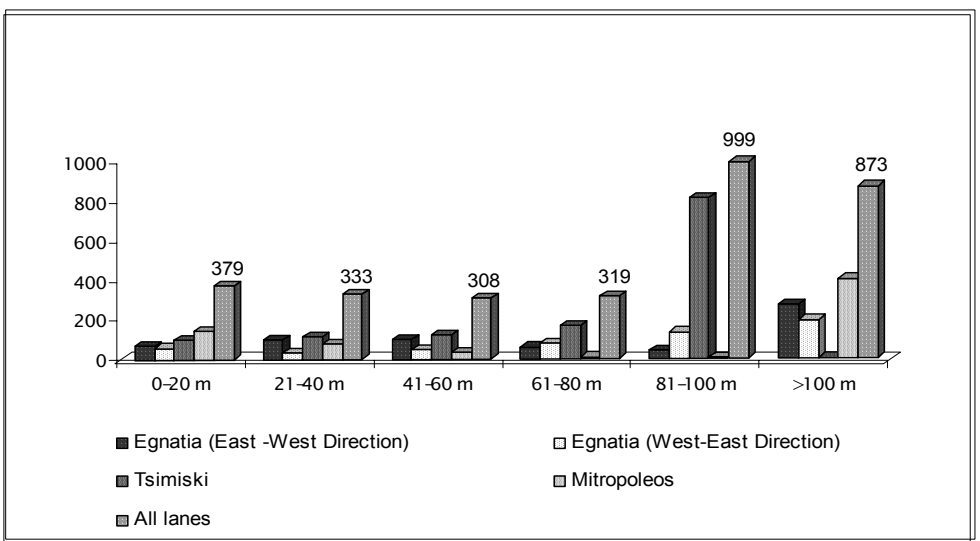

Figure 5: Bus lane violations per length of violation.

\section{Comparison with the results of previous studies}

It is considered interesting to compare the results obtained in this survey with the results obtained in similar surveys carried out in the same area in the past. The first of these surveys was carried out in the framework of the research activities of the Postgraduate Course "Planning, Organization and Management of Transport Systems", Aristotle University of Thessaloniki (AUTh) in May 2005. This survey was carried out in two days (19\& 20 of May 2005) using the same 
methodology as the survey presented in the previous sections. The results obtained as far as the violations per vehicle type are presented in Figure 6 [15]. It must be mentioned at this point that the first graph in Figure 6 corresponds to the situation where drivers of all types of vehicles are considered as possible bus lane violators. The second graph corresponds to the situation where drivers of two-wheel cycles are excluded, since it was assumed that they are entitled to legally enter the bus lanes. The second one of the surveys was carried out by the Hellenic Institute of Transport (HIT) in year 2005 [16]. The results obtained from this survey as far as violations per vehicle type are concerned are presented in Figure 7. It is clear that the results of these two surveys are similar in the case where the drivers of the two-wheel cycles are excluded. The comparison of the previous studies with the December 2005 study has shown that results are similar as far as the situation including drivers of two-wheel cycles is concerned. It arises that the vast majority of violators (in the area of $56 \%$ to $63.8 \%$ ) concerns the drivers of two-wheel drivers. The second category (in the area of $18.2 \%$ to $26 \%$ ) concerns the taxi drivers while the third category (in the area of $14 \%$ to $15.2 \%)$ concerns drivers of passenger cars. It should be mentioned that, taking into account the relatively small number of taxis in the city, the percentage of taxi drivers is high in all surveys. If the comparison is made taking into account the situation without the drivers of the two-wheel cyclists (May 2005 and December 2005 surveys) then results have shown that taxi drivers consist the vast majority (in the area of $50 \%$ to $60 \%$ ) of the bus lane violators (see Figures 2 and 6). The bus lane violations per duration of violation (May 2005 survey) are presented in Figure 8. The comparison of these results with the respective results of the December 2005 survey has shown that in both cases the majority of violations lasts less than $15 \mathrm{sec}$.

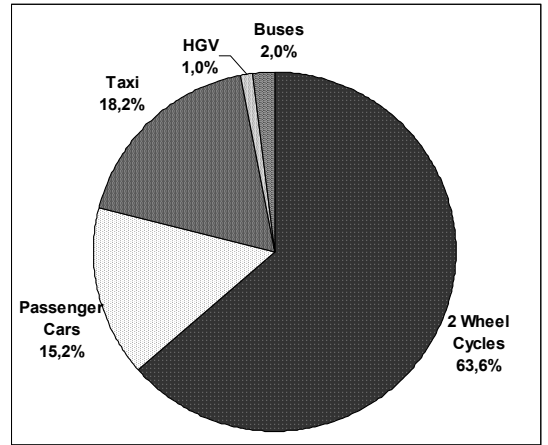

Violations per vehicle type - all types of vehicles

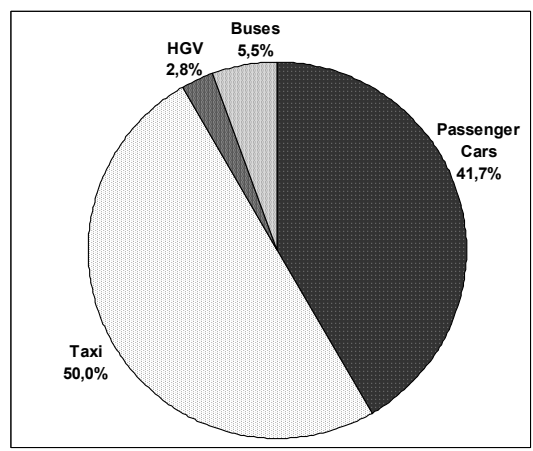

Violations per vehicle type - 2-wheel cycles excluded

Figure 6: Violations per vehicle type (AUTh, 2005). 


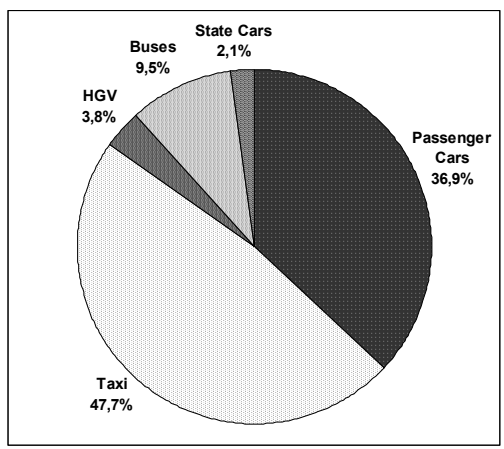

Figure 7: Violations per vehicle type (HIT, 2005).

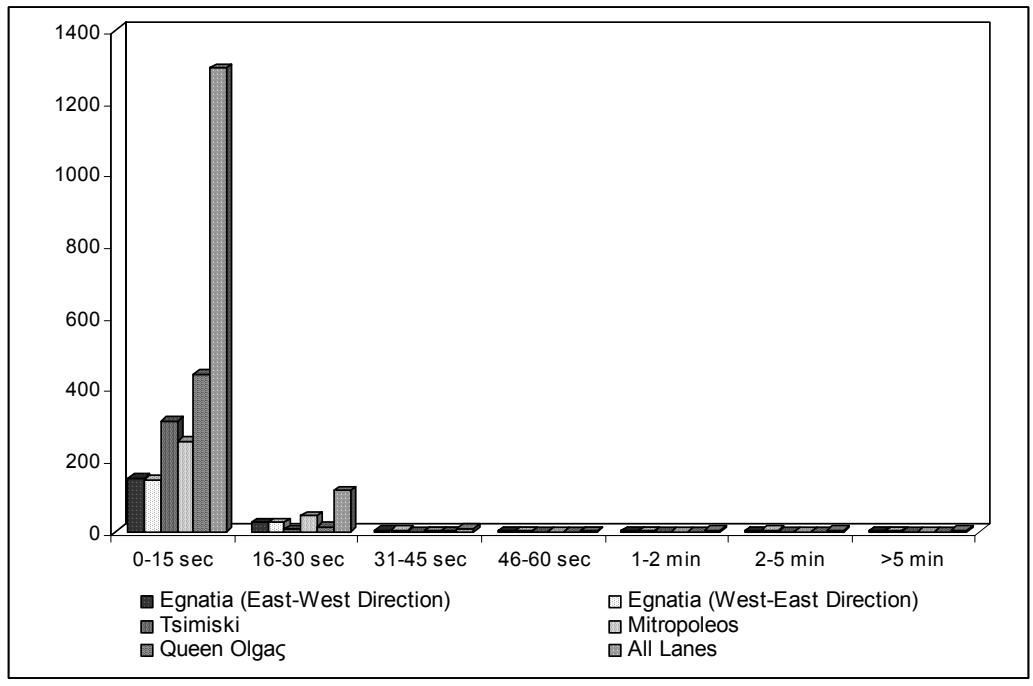

Figure 8: $\quad$ Bus lane violations per duration of violation (AUTh, 2005).

\section{Discussion}

Among the various bus priority measures, bus lanes seem to be the most widespread measure worldwide. The success of this measure strongly depends on the enforcement, something which in turn can be effectively achieved through the use of stationary cameras or bus mounted cameras. The UK experience on this subject is very important as far as the legislation framework and also the technology are concerned. Stationary cameras in the bus lane network of the city of Thessaloniki have been recently implemented in order to strengthen the enforcement of this network. The new Highway Code anticipates five points in the point system and a penalty charge of 200 euros for the violation of bus lanes. These two measures are moving towards the right direction for the improvement of the reliability of the Public Transport system and the overall improvement of 
its performance and attractiveness. The vast majority of violators concern the drivers of the two-wheel cycles followed by the taxi drivers. Another type of drivers like $\mathrm{HGV}$ drivers consist only a small percentage of the bus lane violators. In case that drivers of the two-wheel cycles can legally enter the bus lanes (something which is foreseen in the Highway Code under certain circumstances), then taxi drivers consists more than half of the violators of the bus lane network. These results have shown that enforcement should focus on specific categories of drivers. The experience from other countries should also be considered in the formation of the necessary legislation framework for the support of the operation of the bus lanes network.

\section{References}

[1] Department for Transport, Bus Priority - The Way Ahead, Resource Pack, Edition 2, UK, 2004.

[2] Wiggins A.E. Birmingham bus lane enforcement systems, Proc. of the $9^{\text {th }}$ Int. Conf. on Road Transport Information and Control, pp.80-84, 1998.

[3] JAI, Bus Lane Enforcement, http://www.jai.com/systems/case_show.asp

[4] Department for Transport, Provisional guidance on bus lane (including tramway) enforcement, 2005.

[5] Transportation Alternatives Magazine, Safe Streets. More Lessons from London: Bus Lane Enforcement Cameras, p.16, 2004.

[6] Manchester City Council, Street Management, Bus Lane Enforcement, http://www.manchester.gov.uk/environment/parking/bus

[7] Brighton \& Hove City Council, Bus Lane enforcement, http://www.brighton-hove.gov.uk

[8] Aberdeen City Council, Future Options For Bus Lane Enforcement, Report, 2005.

[9] Roads and Traffic Authority, NSW, Australia, Bus lane cameras, http://www.rta.nsw.gov.au/usingroads/buses/buslane_cameras.html

[10] Chicago Transit Authority (CTA), CTA to Begin Issuing Citations for Bus Lane Scofflaws, CTA Press Release, 2005.

[11] San Francisco Cityscape Forums, Camera Enforcement of Transit Lanes, http://sfcityscape.com

[12] Urban Transport Organisation of Thessaloniki (OASTH), www.oasth.gr

[13] Thessaloniki Police records, 2006.

[14] Department of Transportation \& Hydraulic Engineering, Faculty of Rural \& Surveying Engineering, Aristotle University of Thessaloniki, Examination of bus lane violations in Thessaloniki, Research report, 2005.

[15] Pitsiava-Latinopoulou, M., Basbas, S. \& Andrianos, T. Investigation of enforcement systems for bus lanes, Proc. of the 3rd Int. Conf. on Transport Research in Greece, Hellenic Institute of Transportation Engineers, Hellenic Institute of Transport, pp.242-251, 2006.

[16] Hellenic Institute of Transport, Integrated system for the quality control of the services provided by the Pubic Transport system of Thessaloniki, Report, 2005. 